\title{
Hydrogen Peroxide Sterilization
}

National Cancer Institute

\section{Source}

National Cancer Institute. Hydrogen Peroxide Sterilization. NCI Thesaurus. Code C101689.

A sterilization process that uses hydrogen peroxide to kill microorganisms. 